\title{
Precision radiative corrections to the semileptonic Dalitz plot with angular correlation between polarized decaying and emitted baryons: Effects of the four-body region
}

\author{
J. J. Torres, ${ }^{1}$ M. Neri, ${ }^{2}$ A. Martínez, ${ }^{2}$ A. García, ${ }^{3}$ and Rubén Flores-Mendieta ${ }^{4}$ \\ ${ }^{1}$ Escuela Superior de Cómputo del IPN, \\ Apartado Postal 75-702, México, D.F. 07738, México \\ ${ }^{2}$ Escuela Superior de Física y Matemáticas del IPN, \\ Apartado Postal 75-702, México, D.F. 07738, Mexico \\ ${ }^{3}$ Departamento de Física, Centro de Investigación y de Estudios Avanzados del IPN, \\ Apartado Postal 14-740, México, D.F. 07000, Mexico \\ ${ }^{4}$ Instituto de Física, Universidad Autónoma de San Luis Potosí, \\ Álvaro Obregón 64, Zona Centro, San Luis Potosí, S.L.P. 78000, Mexico
}

(Dated: October 31, 2018)

\begin{abstract}
Analytical radiative corrections of order $(\alpha / \pi)\left(q / M_{1}\right)$ are calculated for the four-body region of the Dalitz plot of baryon semileptonic decays when the $\hat{\mathbf{s}}_{1} \cdot \hat{\mathbf{p}}_{2}$ correlation is present. Once the final result is available, it is possible to exhibit it in terms of the corresponding final result of the three-body region following a set of simple changes in the latter. We cover two cases, a charged and a neutral polarized decaying baryon.
\end{abstract}

PACS numbers: 14.20.Lq, 13.30.Ce, 13.40.Ks 
In this report we shall improve the precision of analytical radiative corrections (RC) to the fourbody region (FBR) of the Dalitz plot of baryon semileptonic decays, $A \rightarrow B l \bar{\nu}_{l}$, with a polarized decaying baryon by including the order $(\alpha / \pi)\left(q / M_{1}\right)$ contributions. The $\hat{\mathbf{s}}_{1} \cdot \hat{\mathbf{p}}_{2}$ angular correlation will be kept explicitly. The corresponding corrections to order $(\alpha / \pi)\left(q / M_{1}\right)^{0}$ were obtained in Ref. [1]. Here we follow the same notation and conventions, and we recall that in this region neither the neutrino nor the real photon can reach zero energy. As explained there, this region must be incorporated into the $\mathrm{RC}$ when bremsstrahlung photons cannot be discriminated either kinematically or by direct detection. This is the case when only the emitted baryon momentum is measured and its corresponding asymmetry coefficient is determined.

The result we want cannot be obtained from the corresponding final result for the three-body region (TBR), because the variable $y_{0}$ plays a double role in the latter case. It appears in the integrand and as the upper limit of the $y=\hat{\mathbf{p}}_{2} \cdot \hat{\mathbf{l}}$ integration variable [see Eqs. (6) and (7) of Ref. [1]]. In the FBR case $y_{0}$ is no more an upper limit of $y$, since now the latter has one as an upper limit, and $y_{0}$ appears only in the integrand. One is then forced to perform the complete calculation. However, once the final result is obtained, it is possible to present it in terms of the final result of the TBR by making simple changes in the latter. This allows us to make a concise presentation and avoid many unnecessary repetitions.

The calculation of RC in the FBR has the same structure as in the TBR, so there is an exact parallelism all along. To mark the difference we shall introduce a subindex $F$ in the expressions and definitions that pertain to the FBR. In this report we shall present only new results, all others will be appropriately referenced.

The complete Dalitz plot without the restriction of kinematically discriminating real photons is

$$
d \Gamma_{i}=d \Gamma_{i}^{\mathrm{TBR}}+d \Gamma_{i}^{\mathrm{FBR}}, \quad i=\mathrm{C}, \mathrm{N}
$$

where $i=\mathrm{C}, \mathrm{N}$ refers to charged and neutral decaying baryon cases, respectively. The analytical RC to order $(\alpha / \pi)\left(q / M_{1}\right)$ to the TBR part including the $\hat{\mathbf{s}}_{1} \cdot \hat{\mathbf{p}}_{2}$ correlation are found in Eqs. (24) and (44) of Ref. [2] for $i=\mathrm{C}, \mathrm{N}$, respectively. The FBR arises only from bremsstrahlung and it can be separated into an unpolarized part $\left(\hat{\mathbf{s}}_{1}=\mathbf{0}\right)$ and into another one containing the $\hat{\mathbf{s}}_{1} \cdot \hat{\mathbf{p}}_{2}$ correlation, that is,

$$
d \Gamma_{i}^{\mathrm{FBR}}=d \Gamma_{i B}^{\prime \mathrm{FBR}}-d \Gamma_{i B}^{(s) \mathrm{FBR}} .
$$

Here the subindex $B$ stresses the bremsstrahlung origin of these parts. To order $(\alpha / \pi)\left(q / M_{1}\right)$ the 
spin-independent part is found, with some changes in notation, in Eq. (32) of Ref. [3] for $i=\mathrm{C}$ and in Eq. (22) of Ref. [4] for $i=\mathrm{N}$. To order $(\alpha / \pi)\left(q / M_{1}\right)^{0}$ the spin-dependent part is found in Eq. (18) of Ref. [1] for $i=\mathrm{C}, \mathrm{N}$.

To obtain the order $(\alpha / \pi)\left(q / M_{1}\right)$ of $d \Gamma_{\mathrm{C} B}^{(s) \text { FBR }}$ we trace a parallelism with Eq. (14) of Ref. [2] for the TBR. The final result is compactly given by

$$
d \Gamma_{\mathrm{C} B}^{(s) \mathrm{FBR}}=\frac{\alpha}{\pi} d \Omega \hat{\mathbf{s}}_{1} \cdot \hat{\mathbf{p}}_{2}\left[B_{2}^{\prime} I_{C 0 F}+C_{A F}^{(s)}\right] .
$$

$B_{2}^{\prime}$ is given in Eq. (7) of Ref. [2]. The infrared convergent $I_{C 0 F}$, explicitly given in Eq. (37) of Ref. [5], corresponds to the infrared divergent $I_{C 0}$ of the TBR. $C_{A F}^{(s)}$ can be arranged as the sum

$$
C_{A F}^{(s)}=C_{I F}+C_{I I F}+C_{I I I F}
$$

where

$$
C_{I F}=\sum_{i=1}^{8} Q_{i+6} \Lambda_{i F}, \quad C_{I I F}=\sum_{i=6}^{15} Q_{i} \Lambda_{(i+3) F}, \quad C_{I I I F}=\sum_{i=16}^{25} Q_{i} \Lambda_{(i+3) F} .
$$

which are the counterparts of Eqs. (21)-(23) of Ref. [2].

The $Q_{i}(i=6, \ldots, 25)$ are quadratic functions of the form factors and are common to both regions. Their explicit expressions are found in Appendix A of Ref. [2]. It should be clear that for $Q_{6}$ and $Q_{7}$ we use $\tilde{Q}_{6}$ and $\tilde{Q}_{7}$ of this Appendix, since the contributions of orders $\left(q / M_{1}\right)^{2}$ and higher have been subtracted.[9]

The analytical form of $C_{A F}^{(s)}$ is obtained by performing explicitly the triple integrals over the real photon variables, contained in the $\Lambda_{i F}$ functions $(i=1, \ldots, 28)$. These integrals result in a set of analytical functions $\theta_{j F}(j=0, \ldots, 22)$. The connection between the $\Lambda_{i F}$ and the $\theta_{j F}$ functions requires several algebraic steps, which are exhibited in terms of intermediate functions $X_{i F}, Y_{i F}$, $Z_{i F}, N_{i F}, I_{F}, \eta_{0 F}, \gamma_{0 F}, \chi_{i j F}$, and $\zeta_{i j F}$.

Once the final results for the FBR are available it is only necessary to give explicitly the $\theta_{j F}$ functions to present it. The $\Lambda_{i F}$ and the intermediate functions[10] can be obtained from the corresponding $\Lambda_{i}$ and $X_{i}, Y_{i}, Z_{i}, N_{i}, I, \eta_{0}, \gamma_{0}, \chi_{i j}$, and $\zeta_{i j}$, respectively, of the TBR by making some simple changes in the latter. This is possible because of the parallelism mentioned above. These changes are: (1) a subindex $F$ is attached to all the corresponding functions of the TBR, (2) the terms proportional to the factor $\left(1-y_{0}\right)$ are replaced by zero, and (3) otherwise the factor $y_{0}$ is kept as such. There are three exceptions to rule (3). In $I, \chi_{11}$, and $\eta_{0}, y_{0}$ appears by itself only once and there it must be replaced by $y_{0}=1$ to produce $I_{F}, \chi_{11 F}$, and $\eta_{0 F}$ respectively. The 
$\Lambda_{i}, X_{i}, Y_{i}, Z_{i}, N_{i}, I, \eta_{0}$, and $\gamma_{0}$ are found in Appendix B of Ref. [2]. It should be stressed that $\Lambda_{2 F}=0$ as is $\Lambda_{2}=0$, and that is why it does not appear in this Appendix. The $\chi_{i j}$, and $\zeta_{i j}$ are found in Sec. IV of Ref. [6], but $\zeta_{10}, \chi_{10}$, and $\chi_{20}$ were not directly given in terms of $\theta_{i}$ there and the above rules cannot be easily applied to them. It is better to give the explicit connection now, namely,

$$
\begin{gathered}
\zeta_{10 F}=p_{2} l\left[\theta_{13 F}-\frac{l}{p_{2}} \theta_{10 F}-\frac{E_{\nu}^{0}}{p_{2}} \theta_{5 F}\right], \\
\chi_{10 F}=l\left[2 \eta_{0 F}-E_{\nu}^{0} \theta_{4 F}-l \theta_{5 F}\right], \\
\chi_{20 F}=2 l^{2}\left[p_{2} y_{0} \theta_{4 F}+E_{\nu}^{0} \theta_{5 F}+2 l \theta_{10 F}-p_{2} \theta_{13 F}-\frac{1}{2} \theta_{14 F}-\frac{E_{\nu}^{0}}{l} \eta_{0 F}\right] .
\end{gathered}
$$

The function $\zeta_{31}$ of Ref. [2] has also a misprint in the term proportional to $\theta_{12}$. In order to avoid further confussions, we provide here the right expression for $\zeta_{31 F}$, which reads

$$
\begin{aligned}
\zeta_{31 F}= & p_{2} l y_{0}\left[2\left(3 E^{2}-l^{2}\right) \theta_{3 F}-6 E^{2}\left(\theta_{4 F}+\beta \theta_{5 F}\right)+\theta_{9 F}\right]-30 l E^{2} p_{2} \theta_{13 F}-30 l^{2} E p_{2} \theta_{19 F} \\
& -\frac{6 l^{3}}{\beta^{4}}\left[5\left(l+\beta E_{\nu}^{0}\right)+3 \beta^{2}\left(p_{2} y_{0}-l\right)\right]\left(\theta_{3 F}-\theta_{4 F}-\beta \theta_{5 F}\right)-18 l^{2} E E_{\nu}^{0}\left(\theta_{4 F}-\theta_{3 F}\right) \\
& +6 p_{2} l^{3} y_{0} \theta_{3 F}+30 l E^{2}\left(l+\beta E_{\nu}^{0}\right) \theta_{10 F}+30 E l^{3} \theta_{20 F}-\frac{1}{2} \theta_{22 F} \\
& -6 p_{2}\left[E^{2} l\left(\beta^{2}-5\right)-\frac{2 l p_{2}^{2}+2 \beta p_{2} l y_{0}\left(E+E_{\nu}^{0}\right)}{b^{+} b^{-}}\right] \theta_{12 F},
\end{aligned}
$$

so that the correct $\zeta_{31}$ of the TBR can be obtained by dropping the subindex $F$ from the $\theta$ 's in the above equation. We need to point out that neither results nor conclusions are affected by that misprint.

Let us illustrate the above procedure with an example. $\Lambda_{15 F}$ is obtained from

$$
\begin{aligned}
\Lambda_{15}= & \frac{\beta\left(E+E_{\nu}^{0}\right)}{4 M_{1}}\left[p_{2}^{2} \theta_{7}+2 p_{2}^{2} E\left(\theta_{4}-\theta_{3}\right)+2 \zeta_{21}-\frac{2}{l} X_{3}\right]+\frac{X_{4}}{2 M_{1}} \\
& -\frac{l^{2} p_{2}}{4 M_{1}}\left(y_{0}^{2}-1\right)+\frac{p_{2}}{4 M_{1} E}\left[4\left(l y_{0}+p_{2}\right) X_{2}+p_{2} \chi_{21}\right],
\end{aligned}
$$

by first putting $y_{0}=1$ into the factor $\left(1-y_{0}\right)$ only and keeping $y_{0}$ as such elsewhere, and second by attaching a subindex $F$ to the intermediate functions. The result is

$$
\begin{aligned}
\Lambda_{15 F}= & \frac{\beta\left(E+E_{\nu}^{0}\right)}{4 M_{1}}\left[p_{2}^{2} \theta_{7 F}+2 p_{2}^{2} E\left(\theta_{4 F}-\theta_{3 F}\right)+2 \zeta_{21 F}-\frac{2}{l} X_{3 F}\right]+\frac{X_{4 F}}{2 M_{1}} \\
& +\frac{p_{2}}{4 M_{1} E}\left[4\left(l y_{0}+p_{2}\right) X_{2 F}+p_{2} \chi_{21 F}\right] .
\end{aligned}
$$

With these changes all we now need is the list of the $\theta_{j F}$ functions. Their explicit forms for $j=$ $1, \ldots, 16$ were already calculated in Ref. [5] and they are found in Appendix B of this reference. $\theta_{0 F}$ is given in Eq. (38) of this reference, $\theta_{1 F}=\theta_{17 F}=0$, and $\theta_{18 F}=1$. 
The functions $\theta_{j F}$ with $j=19, \ldots, 22$ are new. They are defined as $\theta_{19 F}=\int_{-1}^{1} x \xi_{4}^{T}(x) d x$, $\theta_{20 F}=\int_{-1}^{1} x^{3} \xi_{1}^{T}(x) d x, \theta_{21 F}=\int_{-1}^{1} x^{2} \xi_{2}^{T}(x) d x$, and $\theta_{22 F}=\int_{-1}^{1} \xi_{6}^{T}(x) /(1-\beta x) d x$. The functions $\xi_{1}^{T}(x), \xi_{2}^{T}(x)$, and $\xi_{4}^{T}(x)$ are found in the Appendix of Ref. [3]. Here we only need the explicit form of $\xi_{6}^{T}(x)$, which reads

$$
\xi_{6}^{T}(x)=h_{1}(x)(1-\beta x)+\frac{h_{2}(x)}{\left(x+a^{+}\right)\left(x+a^{-}\right)}+\frac{h_{3}^{+}(x)}{\left(x+a^{+}\right)^{2}}+\frac{h_{3}^{-}(x)}{\left(x+a^{-}\right)^{2}}+\frac{h_{4}^{+}(x)}{\left(x+a^{+}\right)}+\frac{h_{4}^{-}(x)}{\left(x+a^{-}\right)},
$$

where

$$
\begin{aligned}
h_{1}(x)= & 24 p_{2} l^{2}\left[\frac{a^{+} y_{0}^{+}}{b^{+}\left(x+a^{+}\right)}+\frac{a^{-} y_{0}^{-}}{b^{-}\left(x+a^{-}\right)}\right] x, \\
h_{2}(x)= & 8 l\left\{6 x\left[2 a_{1}^{2}+p_{2}^{2}-y_{0} p_{2} x a_{1}\right]-p_{2}^{2} x\left(3+x^{2}\right)-3 y_{0} p_{2} a_{1}\left(1+x^{2}\right)+6 x a_{1}^{2}\right. \\
& \left.+6 p_{2}\left[2 y_{0} a_{1}-\left(y_{0}^{2}+1\right) p_{2} x\right]\right\}, \\
h_{3}^{ \pm}(x)= & 2 l\left\{2 p_{2}^{2} x\left(3+x^{2}\right)+6 y_{0} p_{2} a_{1}\left(1+x^{2}\right)-12 x a_{1}^{2} \mp 2 p_{2}^{2} y_{0}\left(y_{0}^{2}+3\right)\right. \\
& \left. \pm 6 a_{1}\left[2 y_{0} a_{1}-\left(y_{0}^{2}+1\right) p_{2} x\right]\right\}, \\
h_{4}^{ \pm}(x)= & \mp\left[\frac{4 l^{2}}{p_{2}}\right]\left[3 p_{2}\left(p_{2} x+y_{0} a_{1}\right)-a^{ \pm}\left(p_{2}^{2} x^{2}+3 y_{0} p_{2} a_{1} x-6 a_{1}^{2}\right)\right] .
\end{aligned}
$$

We have used the definitions

$$
a^{ \pm}=\frac{E_{\nu}^{0} \pm p_{2}}{l}, \quad b^{ \pm}=1+\beta a^{ \pm}, \quad y_{0}^{ \pm}=y_{0} \pm a^{ \pm}, \quad a_{1}=E_{\nu}^{0}+l x
$$

The analytical results of $\theta_{19 F}, \theta_{20 F}, \theta_{21 F}$ and $\theta_{22 F}$ are

$$
\theta_{19 F}=\frac{4}{3 p_{2}}, \quad \theta_{20 F}=\frac{1}{p_{2}}\left(T_{20 F}^{+}+T_{20 F}^{-}\right), \quad \theta_{21 F}=T_{21 F}^{+}+T_{21 F}^{-}, \quad \theta_{22 F}=T_{22 F}^{+}+T_{22 F}^{-},
$$

where

$$
\begin{aligned}
& T_{20 F}^{ \pm}= \pm \frac{1}{4}\left[1-\left(a^{ \pm}\right)^{4}\right] I_{2}^{ \pm} \pm \frac{a^{ \pm}}{2}\left[\left(a^{ \pm}\right)^{2}+\frac{1}{3}\right], \\
& T_{21 F}^{ \pm}=\frac{4}{3} \mp 2 a^{ \pm}\left(y_{0} \pm a^{ \pm}\right)\left[a^{ \pm} I_{2}^{ \pm}-2\right], \\
& T_{22 F}^{ \pm}=\frac{1}{2} L_{0}+\frac{1}{2} L_{1} I_{1}+L_{2}^{ \pm} I_{2}^{ \pm}+L_{3}^{ \pm} I_{3}^{ \pm} .
\end{aligned}
$$

The $I_{1}, I_{2}^{ \pm}$, and $I_{3}^{ \pm}$functions are found at the end of Appendix C of Ref. [6] and the $L_{i}$ are defined 
as

$$
\begin{aligned}
L_{0}= & 48 l^{2} p_{2}\left(\frac{a^{+} y_{0}^{+}}{b^{+}}+\frac{a^{-} y_{0}^{-}}{b^{-}}\right)-16 E\left[p_{2}^{2}+3 l\left(2 l-p_{2} y_{0}\right)\right], \\
L_{1}= & \frac{\beta^{2} h_{2}(1 / \beta)}{b^{+} b^{-}}+\frac{\beta\left[\beta h_{3}^{+}(1 / \beta)+b^{+} h_{4}^{+}(1 / \beta)\right]}{\left(b^{+}\right)^{2}}+\frac{\beta\left[\beta h_{3}^{-}(1 / \beta)+b^{-} h_{4}^{-}(1 / \beta)\right]}{\left(b^{-}\right)^{2}}, \\
L_{2}^{ \pm}= & \frac{-24 p_{2} l^{2}\left(a^{ \pm}\right)^{2} y_{0}^{ \pm}+h_{4}^{ \pm}\left(-a^{ \pm}\right)}{b^{ \pm}}+\frac{b^{ \pm} h_{3}^{ \pm}(0)-h_{3}^{ \pm}\left(-a^{ \pm}\right)}{b^{ \pm} a^{ \pm}}-4 E a^{ \pm}\left[3 l\left(2 l-y_{0} p_{2}\right)-p_{2}^{2}\right] \\
& -\frac{\beta^{2} a^{ \pm} h_{3}^{ \pm}(1 / \beta)}{\left(b^{ \pm}\right)^{2}} \mp \frac{l h_{2}\left(-a^{ \pm}\right)}{2 p_{2} b^{ \pm}}, \\
L_{3}^{ \pm}= & \frac{h_{3}^{ \pm}\left(-a^{ \pm}\right)}{b^{ \pm}}
\end{aligned}
$$

where the functions $h_{i}(x)$ are the same of Eq. (7), but evaluated at the indicated arguments.

For a neutral process we proceed again in parallel to the calculation of $d \Gamma_{\mathrm{N} B}^{(s)}$ given in Sec. IIIB of Ref. [2]. Thus, we can express $d \Gamma_{\mathrm{N} B}^{(s) \text { FBR }}$ as in Eq. (31) of this reference as

$$
d \Gamma_{\mathrm{N} B}^{(s) \mathrm{FBR}}=\frac{\alpha}{\pi} d \Omega \hat{\mathbf{s}}_{1} \cdot \hat{\mathbf{p}}_{2}\left[B_{2}^{\prime} I_{N 0 F}+C_{A F}^{(s)}+C_{N A F}^{(s)}\right]
$$

where $B_{2}^{\prime}$ and $C_{A F}^{(s)}$ are those of Eq. (3). The function $I_{N 0 F}$ corresponds to the infrared divergent $I_{N O}$ of Eq. (35) of Ref. [7]. As in the charged case, $I_{N 0 F}$ is no longer infrared divergent. It can be calculated up to order $\left(q / M_{1}\right)$ by using the approximation $1 / p_{2} \cdot k \simeq 1 / p_{1} \cdot k+q \cdot k /\left(p_{1} \cdot k\right)^{2}$. The result is

$$
I_{N 0 F}=I_{C 0 F}+\delta I_{0}
$$

where $I_{C 0 F}$ is the same of Eq. (3) and

$$
\delta I_{0}=\frac{4 p_{2}}{M_{1} \beta}\left[\frac{y_{0}}{2} \ln \left(\frac{y_{0}+1}{y_{0}-1}\right)-1\right]\left[\left(\frac{1-\beta^{2}}{\beta}\right) \operatorname{arctanh} \beta-1\right] .
$$

It should be remarked that this expression is equal to zero when $\beta \rightarrow 0$ and $y_{0} \rightarrow \infty$.

The other new integrals appear only in $C_{N A F}^{(s)}$. They are

$$
C_{N A F}^{(s)}=D_{3} \rho_{N 3 F}+D_{4} \rho_{N 4 F} .
$$

Here $D_{3}=2\left(f_{1} g_{1}-g_{1}^{2}\right)$ and $D_{4}=2\left(f_{1} g_{1}+g_{1}^{2}\right), \rho_{N 3 F}$ and $\rho_{N 4 F}$ are

$$
\begin{aligned}
& \rho_{N 3 F}=\rho_{I F}+\rho_{I I F}+\rho_{I I I F}, \\
& \rho_{N 4 F}=\rho_{I F}^{\prime}+\rho_{I I F}^{\prime}+\rho_{I I I F}^{\prime} .
\end{aligned}
$$

The analytical forms of the integrals $\rho_{i F}$ and $\rho_{i F}^{\prime}(i=I, I I, I I I)$ can be obtained from Eqs. (34)(39) of Ref. [2] again by changing $y_{0}$ to one as the upper limit in the $y$ integrals. After the 
integration one can see that the analytical form of the $\rho_{i F}$ and $\rho_{i F}^{\prime}$ can be obtained from the analytical expressions of $\rho_{i}$ and $\rho_{i}^{\prime}$ for TBR, which are found in Appendix C of Ref. [2], using the same rules (1), (2), and (3) applied to $\Lambda_{i}$ to obtain the $\Lambda_{i F}$ of the FBR for charged decaying baryons.

We have made crosschecks between numerical integrals and analytical results of the $\Lambda_{i F}, \rho_{i F}$, and $\rho_{i F}^{\prime}$, and they were satisfactory. Our results are model-independent and include terms up to order $(\alpha / \pi)\left(q / M_{1}\right)$, this is as far one can improve the precision of RC before introducing modeldependence into them. They are useful when $l=e^{ \pm}, \mu^{ \pm}$, and $\tau^{ \pm}$. The two cases discussed here, charged and neutral decays, allow one to cover the other four charge assignments predicted by heavy quarks in baryons [8]. When such quarks are involved, the results of Ref. [1] are useful in low statistics experiments (several hundreds of events) and the improved results presented here are useful in medium statistics experiments (several thousands of events).

The authors acknowledge financial support from CONACYT, COFAA-IPN, and FAI-UASLP (Mexico).

[1] R. Flores-Mendieta, A. Garcia, A. Martinez and J. J. Torres, Phys. Rev. D 65, 074002 (2002).

[2] J. J. Torres, R. Flores-Mendieta, M. Neri, A. Martinez and A. Garcia, Phys. Rev. D 70, 093012 (2004).

[3] S. R. Juarez W., Phys. Rev. D 53, 3746 (1996).

[4] S. R. Juarez W., Phys. Rev. D 55, 2889 (1997).

[5] A. Martinez, J. J. Torres, R. Flores-Mendieta and A. Garcia, Phys. Rev. D 63, 014025 (2001).

[6] R. Flores-Mendieta, A. Garcia, A. Martinez and J. J. Torres, Phys. Rev. D 55, 5702 (1997).

[7] A. Martinez, A. Garcia and D. M. Tun, Phys. Rev. D 47, 3984 (1993) [Addendum-ibid. D 50, 2325 (1994)].

[8] A. Martinez, J. J. Torres, A. Garcia and R. Flores-Mendieta, Phys. Rev. D 66, 074014 (2002).

[9] In Ref. [2] $\tilde{Q}_{9}$ actually corresponds to $Q_{9}$ due to the order of approximation implemented.

[10] In Ref. [2] we have detected a misprint in the expression of the intermediate function $\mathcal{J}$, defined at the end of Appendix $\mathrm{D}$ of this reference. In the term proportional to $\theta_{12}$, the denominator of the first summand should read $\beta^{2}$ rather than $\beta$. This misprint, however, does not affect any of the results or conclusions. 